\title{
BIBLIOGRAPHIE.
}

\section{LIVRES NOUVEAUX.}

La première partie du Vol. I de la Bibliotheca Sinica, de M. Henri Cordier, Prix 25 fr., vient de paraître à la librairie E. Guilmoto, successeur de J. Maisonueuve, 6 rue de Mézières, Paris. Elle comprend les colonnes 1-416, e'est-à-dire: I. Ouvrages généraux; II. Gégraphie; III. Noms; IV. Ethnographie et Anthropologie; V. Climat et Metéorologie; VI. Histoire naturelle. La seconde moitié du Vol. J. paraîtra vers le mois de juillet.

La cinquième livraison du Recueil de tectes chinois à l'usage des elèves de l'Eeole spéciale des langues orientales vivantes publié par M. A. VIssière comprend les pages 65 à 80 et les pièces numérotées 93-111 et B 49 - B 57.

Cette Cinquième livraison contient le texte chinois d'une allocution de l'Impératrice douairière (答敕). des conventions de commerce et de frontières franco-chinoises da 20 juin 1895 (conventions Gérard), d'un message du Grand conseil (字寄), de dépêches (次文) des ministères des finances, des rites, des affaires étrangères, d'un ministre chinois en mission à l'étranger (咨呈), d'un rapport au trône émanant du ministère des rites (奏摺); - et de nombreux articles (textes non officiels, dans la partie supérieare des pages) concernant les étudiants chinois en Amérique et ạ Japon, les réformes militaires, les révoltes (Tche-li, Kouang-tong, Kouang-si), le brigandage, le transport des explosifs, le retour de la Cour à Pékin (回 鑾), l'organisation du Bureau des affaires gouvervementales (政務處) 
et du Ministère des affaires étrangères (外務部), des mutations de fonctionnaires, des affaires relatives aux Concessions, aux missionnaires, aux rapports internationaux, budgets étrangers, banques, etc.

Nous arons reçu le Calendrier-Annuaire pour 1904 publié par l'Observatoire de Zi-ka-wei; nous en tirons les renseignements suivants: l'année 1904 comprend la $41^{\mathrm{e}}$ année du $76^{\circ}$ cycle chinois, et lez $29^{\circ}$ et $30^{\ominus}$ années de l'Empereur Kouang-siu. La $41^{\ominus}$ année du $76^{\ominus}$ cycle comprend 354 jours; elle a pour signes cycliques 甲辰 $k^{*} i a-t c h i e n$, correspond au dragon 龍 loung, l'élément est le feu 火 hono. Voici les dates de quelques fêtes: Nouvel an, $1^{\theta}$ lune, $1^{\text {er }}$ jour $=16$ février, $\overrightarrow{\mathrm{\tau}}$ 且 Yuan-tan; Fête des lanternes, $1^{\mathrm{e}}$ lune, $15^{\mathrm{e}}$ jour $=1$ mars, 上元 節 Chang-yuan tsie; Bâteauxdragons, $5^{\mathrm{e}}$ lune, $5^{\ominus}$ jour $=18$ juin 天中節 $T^{\prime}$ ien-tchong tsie.

Le Vol. III des Etudes Diverses de l'ourrage monumental consacré à la Mission Pavie vient de paraitre et il renferme les Recherches sur l'histoire naturelle; l'Anthropologie et la Zoologie sont seules étudiées; les plantes ont été confiées à M. Pierre et leur description aura place dans son grand ouprage; les listes de deux collections géologiques faites par M. Pavie, déterminées la première par M. Fuchs, la seconde par M. Stanislas Mennier, pourront être jointes à une publication postérieure d'un spécialiste. Le volume est orné d'un portrait de M. Pavie et de belles planches en couleurs.

Le Comité de l'Asie française a publié les nouveaux tirages à part suivants des Guides Madrolle: L'art chez les Chinois par R. de Marguerye et Sud de la Chine (Hong-kong, Canton, Macao, le Si-kiang), tirés de la Chine du Sud; le Sud du Yun-nan par Boss D'A tiré de la Chine du Nord; les Voyageurs chinois par Éd. Chavannes, tiré de la Chine du Sud.

Nous avons reȩu de Messrs. Kegan Paul \& Co., la quatrième 
édition de Trübner's Catalogue of Oriental Dictionaries and Grammars qui comprend 154 pages.

Nons remercions M. le Dr. Ernst Kunn de l'envoi du discours important qu'il a prononcé lors de sa prise de possession du rectorat de la Ludwig-Maximilians-Universität, le 21 nov. 1903, à Munich et qui traitait Der Einfluss des Arischen Indiens auf die Nachbarländer im Sïden und Osten.

M. Edlouard Clavery a réuni en brochure la série des intéressants articles sur les Etablissements des Détroits qu'il avait fait paraître dans les Annales coloniales.

Nous avons reçu les Medical Reports des Douanes Impériales Maritimes chinoises pour l'année finissant le 30 sept. 1901; les rapports de Loug-tcheou, Mong-tseu et Sseu-mao sont rédigés en français par les docteurs Gaimard, Georges Barbézieux et Sautarel; ce dernier rapport renferme une observation de lèpre tuberculeuse sur un indigène de 26 ans chez lequel la maladie a débuté à l'âge de 8 ans; sa mère et son oncle maternel étaient lépreux; son père et ses aïeux maternels et paternels n'étaient pas lépreux; la lèpre est excessivement rare à Sseu-mao. Je note dans le rapport de Mongtseu que l'opium fait de grands ravages dans cette ville et que plus du quart de la population est adonné à cette passion. Dans le rapport de Pakhoi, le Dr. J. H. Lowry marque la population étrangère: adultes, hommes 23 , femmes 19; enfants, mâles 8 , féminins, 4.

M. Emile Bourdaret, Ingénieur de la Maison Impériale de Corée a publié deux articles intéressants dans le Bulletin de la Société d'Anthropologie de lyon; le premier a pour titre: I. Note sur les Dolmens de la Corée. II. Les monuments préhistoriques de l'ile de Kang-hoa; le second: Religion et Superstition en Corée. (Séauces du 4 juillet et du 5 déc. 1903.) 